\title{
Multi Beam DOA Estimation Using Robust Convergence Adaptive Algorithm
}

\author{
Y. Murali Krishna ${ }^{1}$, N. Sayedu Khasim ${ }^{2}$ and M. Sreedhar ${ }^{3}$ \\ ${ }^{1,2,3}$ Dept. of Electronics and Communication Engineering, Narasaraopeta \\ Engineering College, Narasaraopeta, AP, India \\ 1yadavalli437@gmail.com, ${ }^{2}$ sayedukhasim@gmail.com
}

\begin{abstract}
Smart Adaptive Array antenna can automatically track the unknown interference signal in real time applications, that it requires to provide nulls towards the interference and offer gain to the required signal to ensure the required signal reception, so it leads the output SINR (signal to Interference and Noise Ratio) is improved. There is a growing need for the development of efficient algorithms for real-time optimization. Several algorithms can be applied for Digital Signal Processors (DSP), which differ in their complexity, convergence and so on

In this paper, an efficient method for the DOA estimation of the linear antenna arrays with the prescribed steering and nulling lobe is presented. The proposed method is based on Least Mean Square (LMS) algorithm; provide a comprehensive and detailed treatment of the signal model used for beam forming, as well as, describing adaptive algorithms to adjust the weights of an array. In order to improve the convergence rate of LMS algorithm in smart antenna system, this paper proposes the GNGD algorithm. By taking advantage of spatial filtering, the proposed scheme promises to reduce the bandwidth required for transmitting data by improving convergence rate. The performance of the GNGD algorithm in the presence of Multi-path effects and multiple users is analyzed using MATLAB simulations. The simulations when compared to that of the LMS algorithm greater improvement in the convergence rate are observed. The results suggest that GNGD algorithm can improve the convergence rate and lead to better system efficiency.
\end{abstract}

Keywords: Smart antenna, LMS, BBNLMS, GNGD, Convergence Rate

\section{Introduction}

The total performance of a communication system depends on interference environment that can be severely degraded if the received signal to interference ratio is unacceptably low. Smart antenna is the critical technique of the third mobile communication, while the core of smart antenna is adaptive algorithm research. Smart antennas can be used for various different benefits. Among those the most important is higher network capacity $[4,5]$ by precise control of signal nulls quality and mitigation of interference combine to frequency reuse reduction distance improving capacity. The term adaptive antenna is used for a phased array when the weight of each element is applied in a dynamic fashion. The amount of weighting on each channel is not fixed at the time of the array design, but rather decided by the system at the time of processing 
the signals to meet required objectives. In other words, the array pattern adapts to the situation and the adaptive process is under control of the system. For example, consider the situation of a communication system operating in the presence of a directional interference operating at the carrier frequency used by the desired signal, and the performance measure is to maximize the output signal to noise ratio (SNR), in such systems the desirable is output SNR should be maximized by cancelling the directional interference using optimal antennas. The antenna pattern in this case has a main beam pointed in the desired signal direction, and has a null in the direction of the interference. Assume that the interference is not stationary but moving slowly. If optimal performance is to be maintained, the antenna pattern needs to adjust so that the null position remains in the moving interference direction.

A system using adaptive antennas adjusts the weighting on each channel with an aim to achieve such a pattern [6]. For adaptive antennas, the conventional antenna pattern concepts of beam width, side lobes, and main beams are not used, as the antenna weights are designed to achieve a set performance criterion such as maximization of the output SNR. On the other hand, in conventional phase-array design these characteristics are specified at the time of design. For adaptive arrays, the conventional antenna pattern concepts of beam width, side lobes and main beams are not used, as the antenna weights are designed to achieve a set performance criterion such as maximization of the output SNR. On the other hand, in conventional phased array design these characteristics are specified at the time of design [7].

In this paper we propose a simple and novel adaptive algorithm for steering the antenna beam electronically. Generally LMS algorithm is widely used in adaptive filter due to its relatively low computational complexity, good stability properties, and relatively good robustness against implementation errors. However, the least mean square (LMS) algorithm has poor convergence rate, which reduces the system performance. In order increase the convergence rate, LMS algorithm is modified by normalization, which is known as normalized LMS (NLMS) [8]. In our work, we adapt Interference (I) NLMS algorithm to increase the convergence rate and reducing computational complexity. Block processing of data in adaptive filters significantly reduces computational burden and improves convergence performance [9]. In literature several smart antenna processing techniques were presented [10-17], to the best of author's knowledge reduction of computational complexity in the array processing is not addressed. Finally in our simulations we considered two received signals and three directions of arrivals (DOAs). Simulation results confirm that the proposed GNGD based beam steering is superior to conventional LMS algorithm in terms of complexity and convergence rate. Normalization of step size in LMS algorithm improves the convergence rate and decreases excess mean square error, by exploiting this we have implemented beam forming using two versions of NLMS algorithms, i.e., Generalized Normalized Gradient Descent (GNGD) algorithm.

\section{Adaptive Beam Forming}

In certain applications the gain of a single antenna may not sufficient, array antennas plays a vital role in such situations. In array antennas the beam can be steered in two ways, i.e., mechanical steering and electronic steering. Adaptive beam forming can be performed in many ways using adaptive algorithms. Several adaptive algorithms are presented in literature. Most of the algorithms are concerned with the maximization of the SNR. A functional diagram of an adaptive array system is shown in Figure 1. 


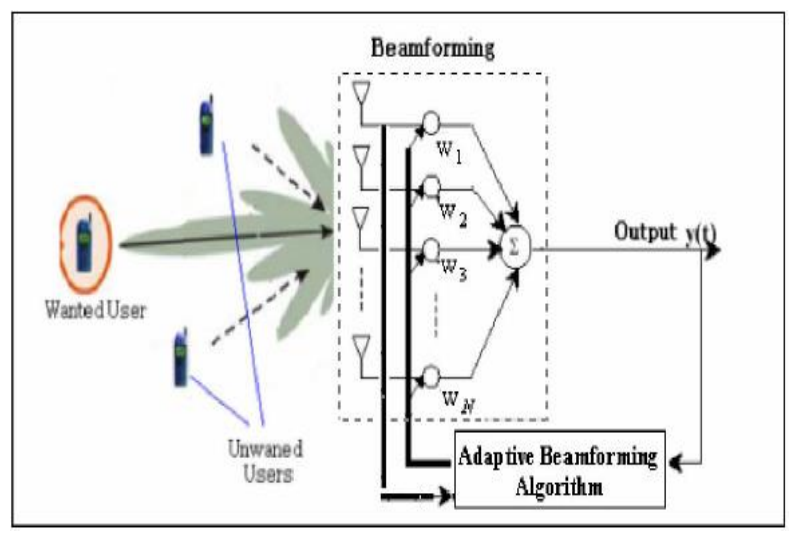

\section{Figure 1. Functional block diagram of an adaptive array system}

Adaptive array systems can locate and track signals (users and interferers) and dynamically adjust the antenna pattern to enhance reception while minimizing interference using signal processing algorithms.

After the system down converts the received signals to baseband and digitizes them, it locates the signal of interest (SOI) using the DOA algorithm, and it continuously tracks the SOI and signal not of interest (SNOI)s by dynamically changing the complex weights (amplitudes and phases of the antenna elements). Basically, the DOA computes the direction-of-arrival of all the signals by computing the time delays between the antenna elements, and afterward, the adaptive algorithm, using a cost function, computes the appropriate weights that result in an optimum radiation pattern. Because adaptive arrays are generally more digital processing intensive and require a complete RF portion of the transceiver behind each antenna element, they tend to be more expensive than switched-beam systems. Adaptive arrays utilize sophisticated signal-processing algorithms to continuously distinguish between desired signals, multipath, and interfering signals, as well as calculate their DOA. This approach updates its transmit strategy continuously based on changes in both the desired and interfering signal locations.

In adaptive beam forming techniques, two main strategies are distinguished. The first one is based on the assumption that part of the desired signal is already known through the use of a training sequence. This known signal is then compared with what is received, and the weights are then adjusted to minimize the Mean Square Error (MSE) between the known and the received signals. In this way, the beam pattern can be adjusted to null the interferers. This approach optimizes the signal-to-interference ratio (SIR), and is applicable to non-line-ofsight (NLOS) environments. Since the weights are updated according to the incoming signals, not only the interference is reduced but the multipath fading is also mitigated. In the second one, the directions of arrivals from all sources transmitting signals to the array antenna are first identified. The complex weights are then adjusted to produce a maximum toward the desired angle and null toward interfering signals. This strategy may turn out to be deficient in practical scenarios where there are too many DOAs due to multi paths, and the algorithms are more likely to fail in properly detecting them. This is more likely to occur in NLOS environments where there are many local scatters close to the users and the base station, thus resulting in a wider spread of the angle of arrival. Another significant advantage of the adaptive antenna systems is the ability to share spectrum. Because of the accurate tracking and robust interference rejection capabilities, multiple users can share the same conventional 
channel within the same cell. System capacity increases through lower inter-cell frequency reuse patterns as well as intra-cell frequency reuse.

\section{Adaptive Algorithms}

An adaptive filter is a transversal filter trained by an adaptive algorithm. The algorithm updates the weights at each iteration by estimating the gradient of the quadratic MSE surface and then moving the weights in the negative direction of the gradient by a minute amount. The constant that determines this amount is referred to as the step size $(\mu)$. When this step size is small enough, the process leads these estimated weights to the optimal weights. The convergence and transient behavior of these weights along with their covariance characterize the LMS algorithm and the way the step size and process of gradient estimation affect these parameters are of great practical importance.

\subsection{The Least Mean Square (LMS) Algorithm:}

Consider a L length LMS based adaptive filter in which ' $\mathrm{W}$ ' is the weight vector updated in accordance with the statistical nature of the input signal $u(n)$ arriving from the antenna array. An adaptive processor will minimize the error $\mathrm{e}(\mathrm{n})$ between a desired signal $\mathrm{d}(\mathrm{n})$ and the array output $y(n)$. LMS is the simplest algorithm used for adaptive processing. This algorithm is based on the knowledge of arriving signal. The knowledge of the received signal eliminates the need for beam forming, but the reference can also be a vector which is somewhat correlated with the received signal. As shown in Figure 1, an adaptive beam former consists of multiple antennas, complex weights, the function of which is to amplify or attenuate and delay the signals from each antenna element, and a summer to add all of the processed signals, in order to tune out the signals not of interest, while enhancing the signal of interest. Hence, beam forming is some time referred to as spatial directions are filtered out, while others are amplified. The output response of the uniform linear array is given by:

$$
y(n)=\widehat{h}^{H}(n) u(n)
$$

We consider the adaptive filter where the input signal $u(n)$ is convolved by an unknown $\mathrm{h}(\mathrm{n})$ filter (to produce $\mathrm{y}(\mathrm{n})$ ) which has an additive interference signal $\mathrm{v}(\mathrm{n})$ before being observed as $d(n)$. The value of error signal estimation is e $(n)=d(n)-y(n)$. The estimated convolved signal $y(n)$ is subtracted from d(n), giving an output signal e(n) containing both the interference $\mathrm{v}(\mathrm{n})$ and a residual signal $\mathrm{r}(\mathrm{n})=\mathrm{y}(\mathrm{n})-\hat{y}(\mathrm{n})$. In many scenarios, such as echo cancellation, the interference $\mathrm{v}(\mathrm{n})$ is actually the signal of interest in the system. We arrive at the recursion for the LMS adaptive algorithm for updating the step as

$$
\mathrm{h}(\mathrm{n})=\mathrm{h}(\mathrm{n}-1)+2 * \mu \mathrm{e}(\mathrm{n}) \mathrm{u}(\mathrm{n})
$$

Where $\mu$ is constant step and the filter taps can be adaptively updated by using above recursive relation.

\subsection{The Normalized Least Mean Square (NLMS) Algorithm}

We may formulate the normalized LMS filter as a natural modification of the ordinary LMS filter. The normalized LMS filter is manifestation of the principle of minimal disturbance, which may be stated that the weight vector of an adaptive filter should be changed in a minimal manner, subject to a constraint imposed on the updated filter's output. An issue of possible concern is that, in overcoming the gradient noise amplification problem 
associated with conventional LMS filter, the Normalized LMS filter introduces a problem of its own, namely that when the tap-input vector $\mathbf{u}(\mathrm{n})$ is small, numerical difficulties may arise because then we have to divide by a small value for the squared norm $\|\mathbf{u}(\mathrm{n})\|^{2}$. To overcome this problem we modify the recursion of weighting factor as

Whereas $\delta>0$

$$
\widehat{\boldsymbol{w}}(\mathrm{n}+1)=\widehat{\boldsymbol{w}}(\mathrm{n})+\frac{\mathfrak{I}}{\left.\partial+\|\mathbf{u}(\mathrm{n})\|^{2}\right]^{2}} \mathbf{u}(\mathrm{n}) \mathrm{e}^{*}(\mathrm{n})
$$

\subsection{Block Based Normalized LMS algorithm}

In a block adaptive filter, the incoming data sequence $u(n)$ is sectioned into L-point blocks by means of a serial to parallel converter, and the blocks of input data so produced are applied to an FIR filter of length $\mathrm{M}$, one block at a time. The tap weights of the filter proceeds on a block-by-block basis rather than on a sample-by-sample basis as in the conventional LMS algorithm Let the following the notation introduced as

$$
\mathrm{u}(\mathrm{n})=[\mathrm{u}(\mathrm{n}), \mathrm{u}(\mathrm{n}-1), \ldots \ldots, \mathrm{u}(\mathrm{n}-\mathrm{M}+1)]^{\mathrm{T}}
$$

Denote the input signal vector at a time $\mathrm{n}$. correspondingly, let

$$
\widehat{\boldsymbol{w}}(\mathrm{n})=\left[\widehat{w}_{0}(\mathrm{n}), \widehat{w}_{1}(\mathrm{n}), \ldots \ldots, \widehat{w}_{\mathrm{M}-1}(\mathrm{n})\right]^{\mathrm{T}}
$$

Denote the tap-weight vector of the filter at a time $n$, The updated weight vector for the number of iterations as

$$
\widehat{\boldsymbol{w}}(\mathrm{n}+1)=\widehat{\boldsymbol{w}}(\mathrm{n})+\frac{\widetilde{\mu}}{p+u(n)_{\max } X(n)_{\max }}
$$

The convergence characteristics are faster than NLMS. Whereas NLMS convergence faster than LMS.

\subsection{The Generalized Normalized Gradient Descent (GNGD) Algorithm}

Gradient-adaptive learning rate algorithms are based on the fact that when the adaptation rate is too small, the gradient tends to keep pointing in the same direction, while if it is too large, the gradient oscillates. Based on the behavior of the stochastic gradient, it is thus possible to infer whether the learning rate must be increased or decreased, and several methods have been proposed in the past to adjust the learning rate based on the gradient [9].

These methods each have a control parameter that is used to determine the learning rate. In the generalized normalized gradient descent (GNGD) algorithm [4], the (normalized) learning rate is

$$
\mu(n)=\frac{\mu_{0}\|u(n)\|^{2}}{\|u(n)\|^{2}+€(n]}
$$

Where $\mu_{0}$ is fixed step size and $€(n)$ is the control parameter. $€(n)$ is recursively calculated as 


$$
€(n)=€(n-1)+\rho \mu_{0} \frac{\Theta(n) e(n-1) u^{T}(n) u(n-1)}{\left(\|u(n)\|^{2}+€(n-1)\right)^{2}}
$$

Because the control parameter is adapted based on the NLMS stochastic gradient behavior, it can only vary relatively slowly (typically requiring tens or hundreds of samples). For that reason, it is important for the optimal learning rate not to depend on rapid changes of the control parameter. The GNGD Algorithm provides more robustness to non-stationary. Under the assumption that $\mathrm{u}(\mathrm{n})$ and $\mathrm{v}(\mathrm{n})$ are zero mean and uncorrelated to each other the theoretical optimal learning rate is equal to the residual-to-error ratio

$$
\mu_{\text {opt }}(n)=\frac{E\left[r^{2}(n)\right]}{E\left[\theta^{2}(n)\right]}
$$

\section{Simulation Results}

To prove the ability of GNGD algorithm in adaptive beam forming we consider two transmitting training signals, each with three multipath components. The second and third multipath of both signals is set to arrive at the base station. This is the case with two white signals and three DOAs. The convergence characteristics of the various algorithms are shown in Figure 2. From this figure it is clear that algorithms converge faster than the conventional LMS algorithms. So that the error amplitude decreases as we are moving from LMS to GNGD algorithm.
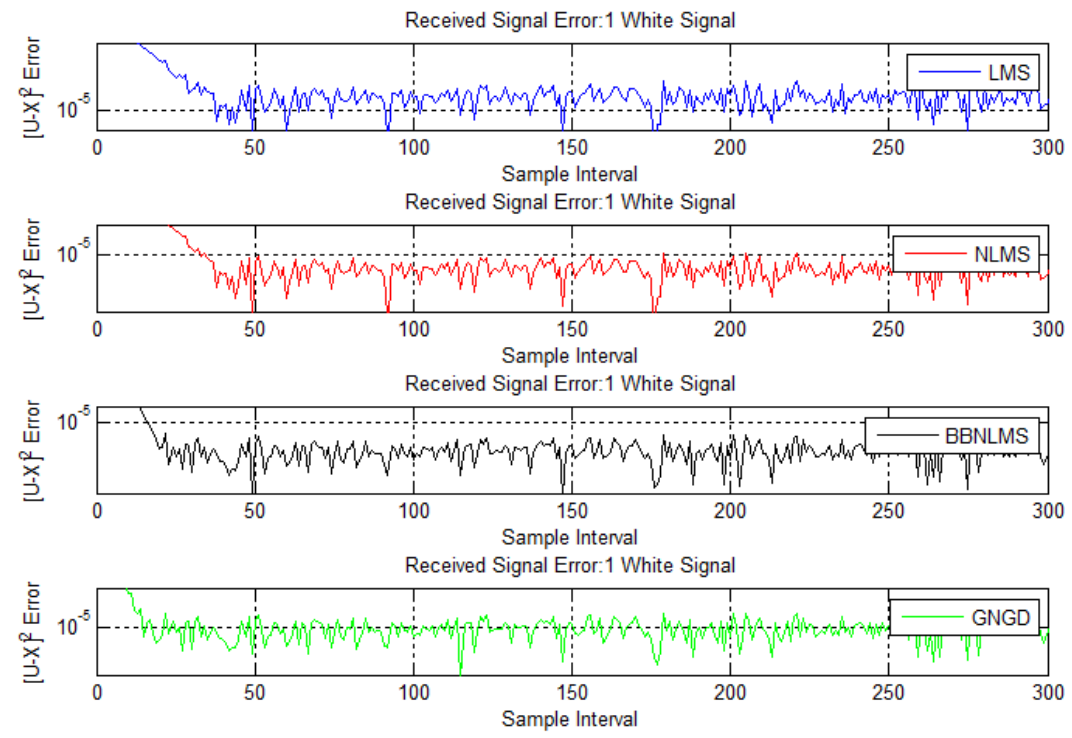

Figure 2. Typical Received signal errors for various algorithms (a).LMS algorithm, (b) NLMS algorithm (c). BBNLMS algorithm, (d). GNGD algorithm 

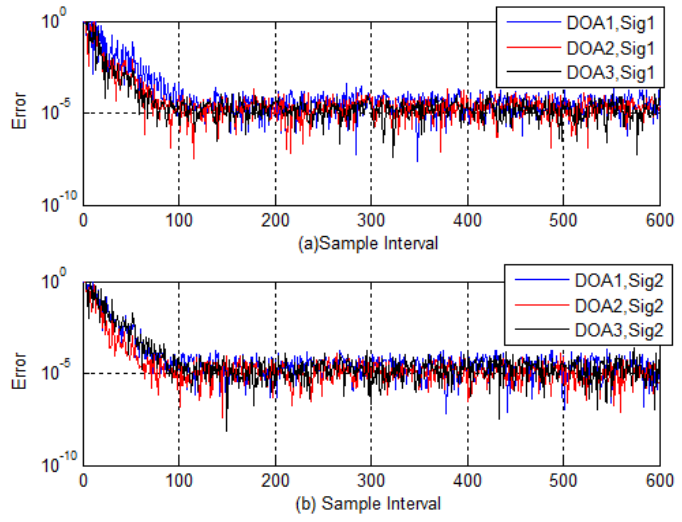

Figure 3. Received signal error with 2 signals and 3DOA's each for LMS algorithm.
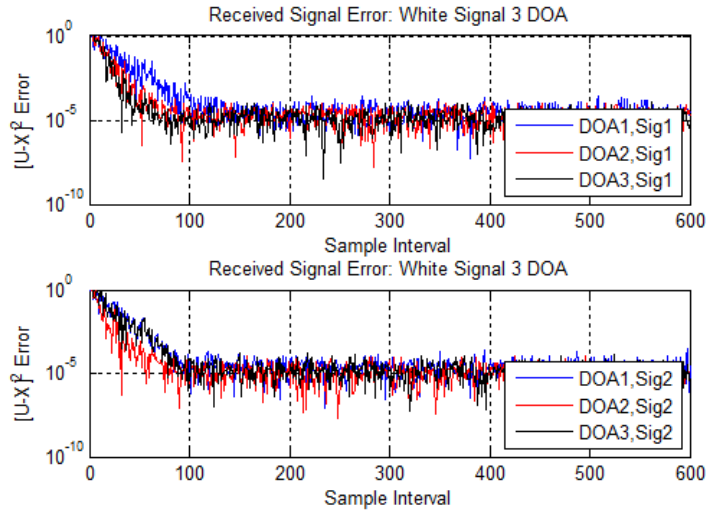

Figure 5. Received signal error with 2 signals and 3DOA's each for NLMS
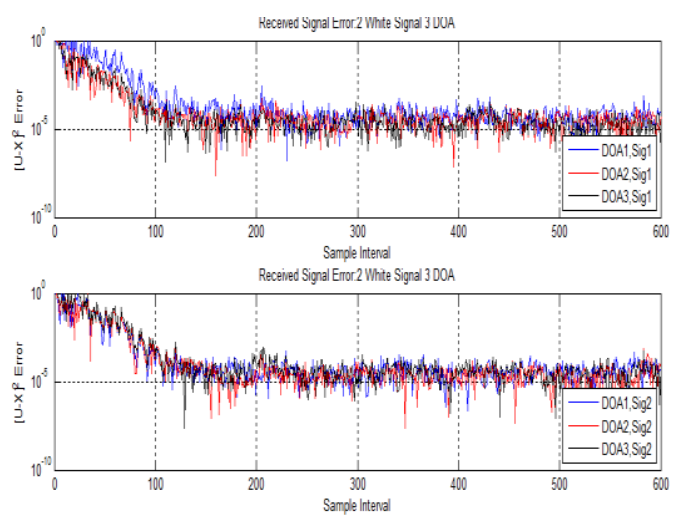

Figure 7. Received signal error with 2 signals and 3DOA's each for BBNLMS algorithm

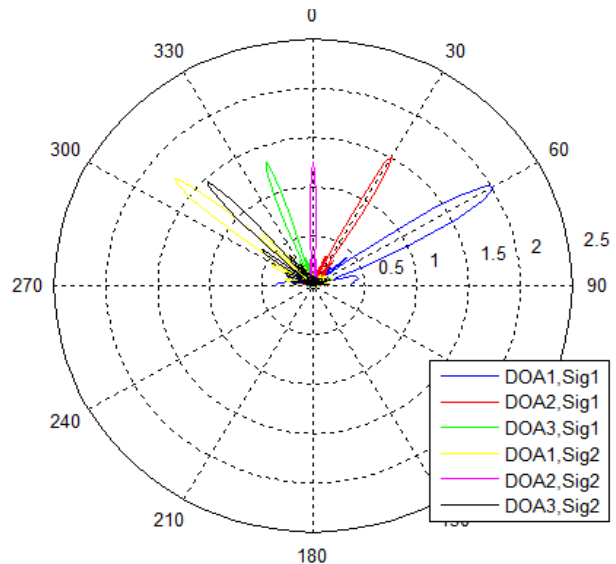

Figure 4. LMS polar plot for 2 signals and 3DOA's each

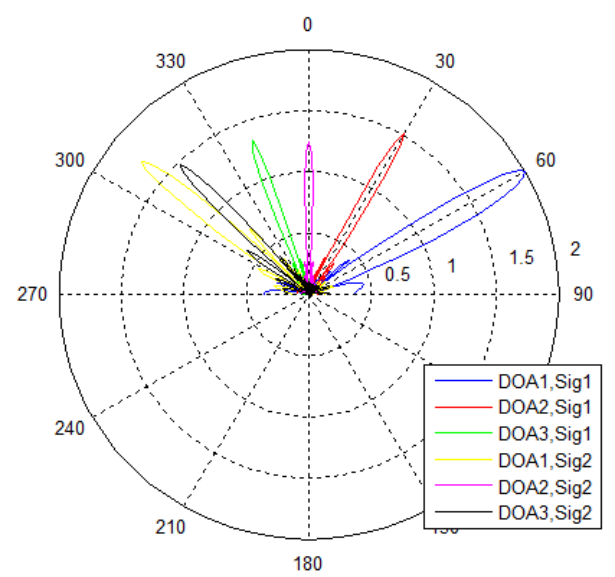

Figure 6. NLMS polar plot for 2 signals and 3DOA's eac

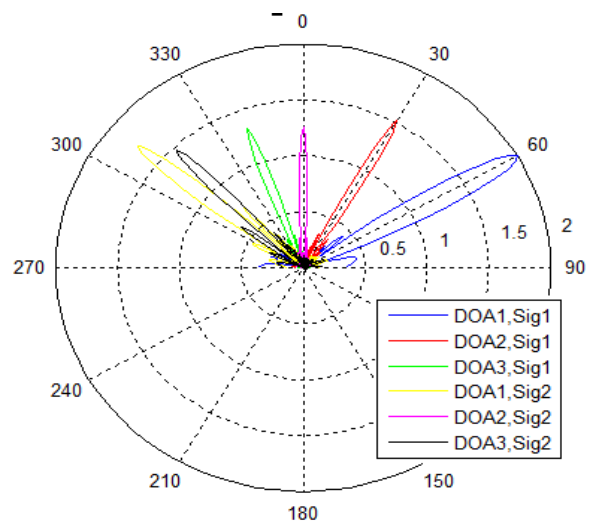

Figure 8. BBNLMS Polar plot for 2 signals and 3DOA's each 

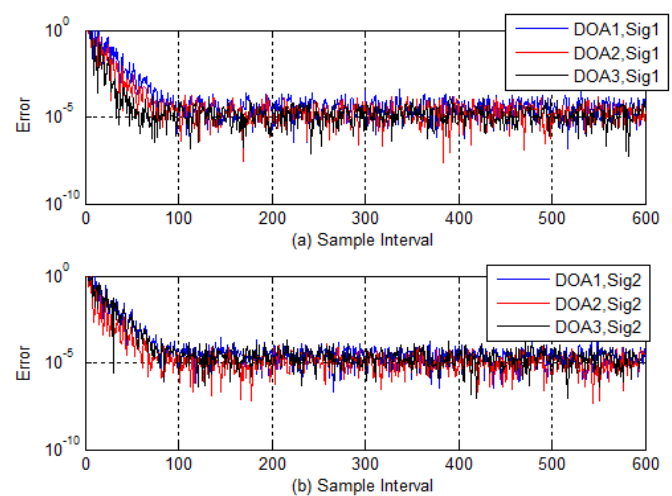

Figure 9. Received signal error with 2 signals and 3DOA's each for GNGD algorithm.

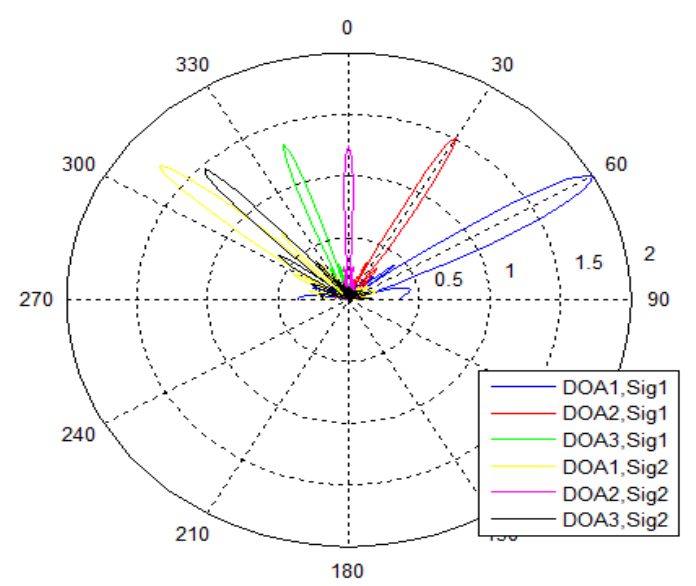

Figure 10. GNGD: Polar plot for 2 signals and 3DOA's each

By keen observation of Figure 3 we can say that convergence starts for LMS approximately after 40th sample, whereas for NLMS and BBNLMS algorithm it is approximately after $30^{\text {th }}$, 25th sample, superiorly for GNGD convergence starts approximately after 20th sample. In addition to that the proposed algorithm takes quick stabilized state compared to the other algorithms. In our simulations we have considered the case of two received signals with three DOAs. The transmission of two different signals with one DOA each is in effect the same as sending one signal with two multipath separated by at least one sample period. This is because in both situations the two signals are uncorrelated with each other. The $1^{\text {st }}$ Signal is exposed to gain with amplitude 2 and the $2^{\text {nd }}$ signal 1.5 and $3^{\text {rd }}$ may be at 1.2 .These results vary to a certain degree, when compared to the GNGD algorithm shown in Figure 10 so finally the GNGD algorithm is producing better performance as compared to remaining algorithm which are discussed in this paper.

\section{Conclusion}

In this paper several beam forming algorithms are presented with some simulations and results. Using GNGD, compared to LMS algorithm, a 50\% increase in overall convergence rate is achieved in a multi user multipath environment. With regards to beam patterns, GNGD algorithms are able to steer beams in the direction of the desired signal and place nulls elsewhere.

\section{References}

[1] G. S. N. Raju, “Antennas and Wave propagation”, Pearson International Publications, Singapore, (2010).

[2] R. E. Collin and F. J. Zucker, “Antenna Theory”, Mc-Graw Hill, New York, (1969).

[3] R. E. Collin, "Antennas and Radiowave Propagation", Mc-Graw Hill, New York, (1985).

[4] C. B. Dietrich Jr., W. L. Stutzman, B. -K. Kim and K. Dietze, "Smart Antennas in Wireless Communications: Base- Station Diversity and Handset Beam forming", lEEE Antennas and Propagation Magazine, vol. 42, no. $5,(2000)$ October.

[5] M. Chryssomallis, "Smart Antennas", lEEE Antennas and Propagation Magazine, vol. 42, no. 3, (2000) June.

[6] F. Gross, "Smart Antenna For Wireless Communication", Mcgraw-hill, (2005) September.

[7] A. Alexiou and M. Haardt, "Smart Antenna Technologies for Future Wireless Systems: Trends and Challenges”, IEEE Communication Magazine, vol. 42, no. 9, (2004) September, pp. 90-97. 
[8] B. Widrow and S. D. Stearns, "Adaptive Signal Processing", Pearson Education Asia, Second Indian Reprint, (2002).

[9] S. Haykin, "Adaptive filter theory", Fourth edition, Pearson Education, Asia, (2002).

[10] S. D. Blostein and H. Leib, "Multiple antenna systems: Role and impact in future wireless access", IEEE Comm. Mag., vol. 41, no. 7, (2003) July, pp. 94-101.

[11] J. H. Winters, "Smart antennas for wireless systems", IEEE Personal Comm., vol. 5, no. 1, (1998) February, pp. 23-27.

[12] A. J. Paulraj, D. Gesbert and C. Papadias, "Smart antennas for mobile communications", Encyclopedia for Electrical Engineering, John Wiley Publishing Co., (2000), pp. 1-15.

[13] V. Kalinichev, "Analysis of beam-steering and directive characteristics of adaptive antenna arrays for mobile communications", IEEE Antennas Propagat. Mag., vol. 43, no. 3, (2001) June.

[14] E. Charpentier and J. Laurin, "An implementation of a directionfinding antenna for mobile communications using a neural network", IEEE Trans. Antennas Propagat., vol. 47, no. 7, (1999) July.

[15] M. S. Choi, G. Grosskopf, D. Rodhe, B. Kuhlow, G. Przyrembel and H. Ehlers, "Experiments on DOAestimation and beamforming for $60 \mathrm{GHz}$ smart antennas", Proc. Vehicular Technology Conference, 2003 (VTC 2003-Spring), vol. 2, (2003) April 21-24, Jeju, Korea, pp. 1041-1045.

[16] K. Kyungjunq, T. K. Sarkar, H. Wang and M. Salazar-Palma, "Direction of arrival estimation based on temporal and spatial processing using a direct data domain (D3) approach", IEEE Trans.Antennas Propagat., vol. 52, no. 2, (2004) February, pp. 533-541.

[17] M. Pastorino and A. Randazzo, "A New Smart Antenna for the Estimation of Impinging Signals in Wireless Communications", International Conference on Antenna Technologies (ICAT-2005), Ahmedabad, India, (2005) February 21-22.

[18] M. Z. Rahman, V. Kumar and G. V. S. A. Karthik, "A low complex adaptive algorithm for antenna beam steering", Signal Processing, Communication, Computing and Networking Technologies (ICSCCN), 2011 International Conference, (2011) July 21-22, pp. 317-321.

\section{Authors}

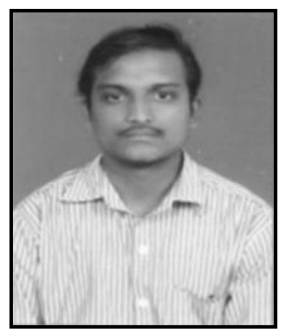

\section{Y. Murali Krishna}

He received his M.Tech degree from JNTUK, A, P, India in 2011. Presently he is working as a Assistant professor, in Department of Electronics and Communication Engineering, Narasaraopeta Engineering College, Guntur, A.P, India. His current areas of research interests include Communication and Radar systems, Smart antennas.

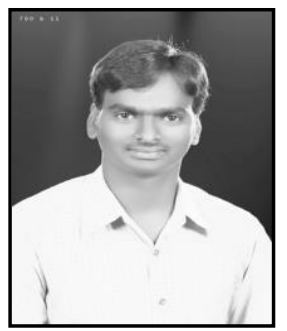

\section{N. Sayedu khasim}

He received his M.Tech degree from ANU, A, P, India in 2010. Presently he is working as a Assistant professor, in Department of Electronics and Communication Engineering, Narasaraopeta Engineering College, Guntur, A.P, India. His current areas of research interests include Communication and Radar systems, Digital signal processing. 
International Journal of Hybrid Information Technology Vol.6, No.6 (2013) 